\title{
Les associations de parents d'enfants autistes
}

> Si dans les années 1970 les associations de parents d'enfants autistes réclamaient l'ouver-

\section{De nouvelles orientations}

Brigitte Chamak ture d'hôpitaux de jour, dans les années 1990, cette revendication s'est transformée en une remise en cause des professionnels et une volonté de s'éloigner du milieu psychiatrique. Longtemps mises en accusation, les familles se montrent de plus en plus sceptiques vis-à-vis des autorités médicales et administratives et rejettent parfois les prises en charge proposées par les institutions. Elles réclament un diagnostic précoce pour obtenir des aides et mettre en place des interventions adaptées. La psychanalyse, qui pendant longtemps a constitué l'approche privilégiée en matière d'autisme, est aujourd'hui décriée par les associations qui réclament l'intégration scolaire pour leurs enfants et la généralisation des méthodes éducatives et comportementales. <

Depuis les années 1990, les représentations de l'autisme ont connu de profondes transformations à la suite de l'élargissement des critères diagnostiques introduit dans les classifications américaines et internationales des maladies [1, 2]. Autrefois considérée comme une maladie rare, sévère et incurable, l'autisme est devenu, avec la création de la catégorie des troubles envahissants du développement (TED), un syndrome incluant aussi bien des sujets sans langage que des personnes avec des capacités langagières mais des difficultés d'interactions sociales et des intérêts restreints. Les associations de parents d'enfants autistes ont intégré très rapidement ces transformations, plus rapidement que ne l'ont fait les professionnels, qui éprouvent des réticences à enfermer l'enfant dans une catégorie et à poser un diagnostic qui peut être lourd de conséquences. Cette réserve, qui résulte des représentations très négatives attachées à l'autisme, met souvent les parents dans l'embarras car si leur enfant n'est pas pris en charge à plein temps dans un hôpital de jour ou un institut médico-social, ils doivent faire état d'un diagnostic pour obtenir une aide financière et trouver des interventions adaptées.

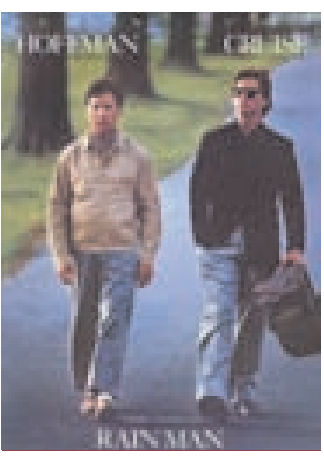

CESAMES - Centre de Recherche Psychotropes, Santé Mentale, Société, CNRS (UMR 8136), Inserm (U611), Université Paris Descartes, 45 , rue des Saints-Pères, 75270 Paris Cedex 06, France. brigitte.chamak@ paris5.sorbonne.fr

En France, le décalage entre les représentations des parents et celles des professionnels est à l'origine d'un fossé qui a du mal à se combler. Pour mieux comprendre ce phénomène, nous avons entrepris de retracer la dynamique historique du mouvement des associations de parents et son impact sur les politiques publiques. Étudier cette mobilisation constitue un moyen pour analyser la nature du travail politique à la fois à l'intérieur de ces groupes et en direction de l'extérieur (média, opinion publique, politiques, professionnels). Créer des associations est, pour les familles, un moyen de faire entendre leur voix et de prendre des initiatives $[3,4]$, mais c'est aussi un moyen pour intervenir en tant que médiateur institutionnel reconnu par les pouvoirs publics, les autres associations et les institutions scientifiques et médicales.

Depuis les années 1980, la forme associative est apparue comme le support privilégié des demandes et des contestations [5]. Elle représente, en outre, pour ses acteurs, un moyen important de promotion sociopolitique. Martine Barthélémy [6,7] a décrit ce qui, pendant les années 1990, a nourri le recours aux associations: à la réduction de la demande d'État mise en avant dans les années 1970, a succédé la demande d'un autre État, qui s'appuie sur l'initiative et la mobilisation des acteurs locaux, entreprises, élus et associations. Les pouvoirs publics prescrivent ainsi aux associations un rôle de traduction et d'encadrement des demandes sociales et de responsabilisation des citoyens. 


\section{Dynamique historique de la mobilisation des associations de parents}

Trois générations d'associations de parents ont pu être identifiées [2]. La première association, L'ASITP (Association au service des personnes inadaptées ayant des troubles de la personnalité), qui changera de nom pour devenir Sésame Autisme, a lutté en premier lieu contre le manque de structures en créant en 1963 le premier hôpital de jour pour enfants, afin de leur éviter la séparation d'avec leurs parents. Au milieu des années 1980, une deuxième génération d'associations a vu le jour: d'une part, I'ARAPI (Association pour la recherche sur l'autisme et les psychoses infantiles $)^{1}$, réunissant parents et professionnels pour favoriser la recherche sur l'autisme, et, d'autre part, des associations, Pro Aid Autisme et AIDERA, qui ont créé des structures spécialisées utilisant l'approche Teacch (Treatment and education of autistic and related communication handicapped children). Élaboré en 1966 par Eric Schopler, en Caroline du Nord, ce programme proposait un projet d'éducation spéciale mettant l'accent sur les capacités émergentes de l'enfant et la collaboration entre les parents et les professionnels [8].

La troisième génération d'associations, beaucoup plus revendicative, est apparue en 1989 avec la création d'Autisme France à la suite de la scission qui s'est opérée au sein de l'ASITP entre ceux qui continuaient à vouloir travailler en collaboration avec les psychiatres et ceux qui voulaient rompre avec la psychiatrie et les approches psychanalytiques de l'autisme, les jugeant trop culpabilisatrices vis-à-vis des parents et inefficaces pour les enfants. Cette dernière génération d'associations est résolument orientée vers les méthodes éducatives et comportementales et l'intégration scolaire. À l'image des groupes de consommateurs, elle cherche à se constituer en communauté active pour rééquilibrer les rapports de force afin que les familles ne se retrouvent plus soumises et impuissantes face aux psychiatres. C'est le cas de l'association Lea Pour Samy, créée en 2001.

Dans le contexte de la généralisation des valeurs de l'autonomie à l'ensemble de la vie sociale, le mouvement des associations de parents a pris de l'ampleur. Ce contexte se caractérise par l'ancrage dans la vie quotidienne d'un double idéal de réalisation de soi et d'initiative individuelle, c'est-à-dire la capacité à décider et à agir par soi-même [9]. Ayant accès, par l'intermédiaire d'Internet, aux informations médicales et scientifiques, ces associations acquièrent une capacité d'expertise qui modifie leurs relations au monde médical et remettent en cause le modèle asymétrique où le psychiatre est celui qui décide. Des parents qui, autrefois, subissaient les décisions et les contraintes imposées par le milieu médical se refusent aujourd'hui à être tenus à l'écart et réclament le choix des thérapies pour leur enfant. Ils insistent sur l'intégration scolaire et se réfèrent au modèle nord-américain de l'autisme, considéré comme un exemple de modernité et d'innovation. En se référant aux classifications et publications internationales, ils contribuent à modifier les frontières entre expertises professionnelles et profanes et donnent ainsi davantage de crédit à leurs revendications.

${ }^{1}$ L'ARAPi a changé d'intitulé en 1995 pour devenir l'Association pour la recherche sur l'autisme et les préventions des inadaptations.

\section{Impact sur les politiques publiques}

La pression des associations a donné lieu à une mobilisation des pouvoirs publics qui a conduit à la Circulaire Veil de 1995 définissant un plan d'action pour cinq ans afin d'améliorer la prise en charge de l'autisme, et à la loi Chossy de 1996 reconnaissant l'autisme comme un handicap. Forte demande des associations de parents, cette reconnaissance est perçue comme un moyen de s'éloigner de la psychiatrie, de bénéficier d'aides financières et d'une intégration scolaire pour leur enfant grâce à la loi du 11 février 2005 «pour l'égalité des droits et des chances, la participation et la citoyenneté des personnes handicapées ». Cette loi pose, en effet, le principe de l'inscription de tout enfant porteur de handicap dans l'établissement scolaire le plus proche du domicile. Mais, l'inscription d'un enfant à l'école ne signifie pas forcément qu'il puisse bénéficier d'interventions adaptées si les moyens matériels ne sont pas disponibles et si les personnels n'ont pas reçu une formation spécialisée.

Si dans les années 1970 les associations de parents réclamaient l'ouverture des hôpitaux de jour, dans les années 1990, cette revendication s'est transformée en une remise en cause des professionnels et une volonté de s'éloigner du milieu psychiatrique. On peut constater une convergence entre les demandes des nouvelles associations et les politiques gouvernementales visant à réduire le nombre de lits d'hospitalisation. Cette convergence n'est pourtant qu'apparente puisque les objectifs ne sont pas les mêmes. Pour les parents, il s'agit de stimuler une politique d'intégration scolaire avec des professionnels formés et des classes à petits effectifs alors que, pour les pouvoirs publics, il s'agit de réaliser des économies : les orientations proposées vont dans le sens d'un désinvestissement vis-à-vis des établissements publics et de structures pérennes au profit de l'aide aux initiatives privées. Le contrôle de ces nouvelles structures par l'État est assuré par un soutien financier minimal conditionné par une accréditation. Ainsi, l'apparente convergence correspond davantage à une instrumentalisation, par les pouvoirs publics, des tensions entre les associations de parents et les professionnels.

\section{Impact sur les réorganisations professionnelles}

Les associations de parents ont gagné la bataille médiatique et ont réussi à obtenir certains changements mais elles sont confrontées aux résistances des professionnels qui sont souvent hostiles aux méthodes comportementales et restent attachés à la fois à une conception restreinte de l'autisme (refusant l'élargissement des critères diagnostiques) et aux approches d'inspiration psychanalytique. 
Des recompositions professionnelles sont pourtant à l'œuvre. Le nombre de psychiatres et d'infirmiers diminue, celui des psychologues, des orthophonistes et des psychomotriciens augmente. Grâce au soutien des associations de parents, les psychologues qui pratiquent les méthodes éducatives et comportementales peuvent se créer une clientèle, acquérir davantage d'autonomie et s'affranchir de la tutelle des psychiatres. Les alliances entre psychologues cognitivo-comportementalistes et associations de parents d'enfants autistes contribuent à renforcer ce courant professionnel qui, malgré l'hostilité des autres psychologues, connaît succès et reconnaissance publique. Les organismes de recherche jouent également un rôle important dans cette recomposition professionnelle. Or, les associations y étendent leur influence, notamment à l'Inserm, qui favorise l'implication des associations dans la recherche médicale [10]. Dans le cadre du Plan santé mentale mis en place en 2001, l'Inserm a été sollicité pour établir un état des lieux de la littérature internationale concernant les différentes approches psychothérapeutiques. En 2004, cet institut publiait un rapport d'expertise où les thérapies cognitivocomportementales y apparaissaient comme globalement plus efficaces que les approches psychodynamiques et systémiques. Dans le cas de l'autisme, la méthode ABA (Applied Behavior Analysis), fondée sur le principe comportemental de conditionnement opérant, est nettement valorisée. Une étude de Lovaas [11] est citée et la conclusion est que: « 6 ans après la fin du traitement, $42 \%$ des enfants ne se distinguent plus des autres enfants », sans préciser que ceux qui ont le plus progressé avaient les meilleures compétences cognitives au départ et que, comparé à d'autres études, l'âge mental initial y est supérieur [12].

\section{Conclusion}

L'élargissement des critères diagnostiques et le développement des méthodes éducatives et comportementales proposées par le modèle américain de l'autisme ont permis aux parents d'enfants autistes de trouver une alternative à l'ancien modèle qui stigmatisait à la fois les parents, cultivant leur sentiment de culpabilité, et les personnes autistes, qui étaient considérées comme des psychotiques sévèrement atteints ou des retardés mentaux. L'augmentation du nombre de personnes concernées, liée à la nouvelle définition élargie de l'autisme et à une détection plus fréquente et plus précoce, a permis aux associations de parents d'ériger l'autisme en problème de santé publique et d'interpeller les pouvoirs publics. L'augmentation de la prévalence a eu également pour effet de transformer l'autisme en une source potentielle de marchés: formations diverses, ouvrages en tous genres, régimes alimentaires spéciaux (sans gluten, sans caséine), tests génétiques [13], dosages biologiques, et multiples thérapies plus ou moins orthodoxes. Les familles se trouvent dans la situation de proies faciles à exploiter, prêtes à tout pour aider leur enfant. Elles contribuent à l'essor du marché des méthodes éducatives et comportementales, qui inclut des formations pour les parents et les professionnels, et prennent position dans les controverses scientifiques. La relation de confiance entre parents et professionnels s'est nettement dégradée. Suspicion et manque de confiance se généralisent chez les parents et entravent une alliance thérapeutique indispensable pour élaborer un projet à long terme pour l'enfant. De mauvaises pratiques du côté des professionnels et des réactions agressives de la part de certaines familles ou associations ont abouti à la situation actuelle en France, où les parents préfèrent l'intégration scolaire à tout prix plutôt qu'une prise en charge en hôpital de jour ou en IME (Institut médico-éducatif). Tandis que les associations de parents contribuent à façonner les politiques publiques et favorisent l'orientation vers des méthodes de prise en charge éducatives et comportementales, les associations de personnes autistes réclament d'être reconnues comme des partenaires à part entière et refusent le statut de malade ou d'handicapé. Elles redéfinissent l'autisme comme une différence, un autre mode de fonctionnement cognitif. Pour elles, il ne s'agit pas seulement de défendre des intérêts mais d'en appeler à de nouvelles formes d'organisation de la société dans laquelle les différences ne seraient plus considérées comme des écarts à la norme, mais comme des formes d'identité à part entière [14]. $\diamond$ Autism and social movements: French parents' associations and international autistic individuals' organizations

\section{RÉFÉRENCES}

1. Chamak B, Cohen D. L'autisme : vers une nécessaire révolution culturelle. Med Sci (Paris) $2003 ; 19: 1152-9$.

2. Chamak B. Les transformations des représentations de l'autisme et de sa prise en charge : le rôle des associations en France. In : Nouveau malaise dans la civilisation : regards sociologiques sur la santé mentale, la souffrance psychique et la psychologisation. Université du Québec de Montréal: Cahiers de recherche sociologique, 2005 ; n 41 : 171-92.

3. Barral C, Paterson F, Stiker HJ, Chauvière M. L'institution du handicap: le rôle des associations XIXe-XXe siècles. Rennes: Presses Universitaires de Rennes, 2000.

4. Paterson F, Barral C. L'Association Française contre les Myopathies: trajectoire d'une association d'usagers et construction associative d'une maladie. Sciences Sociales et Santé 1994 ; XII : 79-111.

5. Théry $H$. La place et le rôle du secteur associatif dans le développement de la politique d'action sanitaire et sociale, avis et rapport du Conseil économique et social, juin 1986.

6. Barthélémy M. Associations : un nouvel âge de la participation? Paris : Presses de Sciences Politiques, $2000: 286 \mathrm{p}$.

7. Barthélémy M. Les associations et la démocratie : la singularité française. In : Qu'est-ce que la société ? Université de tous les savoirs, vol. 3. Paris: Odile Jacob, 2000.

8. Schopler $\varepsilon$, Lansing M, Reichle RJ. Stratégies éducatives de l'autisme et des autres troubles du développement. Paris : Masson, 2003.

9. Ehrenberg A. Agir de soi-même Esprit 2005 ; juillet : 200 -9.

10. Barbot J. Les malades en mouvements. Paris: Balland, 2002.

11. Lovaas 01 . Behavioral treatment and normal education and intellectual functioning in young autistic children. J Consult Clin Psychol 1987 ; 55 : 3-9.

12. Smith T. Outcome of early intervention for children with autism. Clinical Psychology Science and Practice $1999 ; 6: 33-49$.

13. Jordan B. Chroniques génomiques : demandez le test de l'autisme! Med Sci (Paris) 2005 ; $21: 886-7$.

14. Chamak B. Autism and social movements: French parents' associations and international autistic individuals' organizations. Sociology Health Illness 2008 ; $30: 76-96$.

\section{TIRÉS À PART}

B. Chamak 